\title{
A UNIFIED COMPUTER MODEL OF THE SPRAY FORMING PROCESS OF INCONEL 718 RINGS
}

\author{
Iñaki Garmendia ${ }^{1}$, Aitor Landaberea ${ }^{1}$, Teresa Vicario ${ }^{1}$, Udo Fritsching ${ }^{2}$, Omar Belkessam², \\ Patrick S. Grant ${ }^{3}$, Jiawei $\mathrm{Mi}^{3}$ \\ ${ }^{1}$ Inasmet Research Centre; Mikeletegi 2 ; 20009 San Sebastián, Spain \\ ${ }^{2}$ University of Bremen, SFB 372 ; Badgasteiner Str. 3; 28359 Bremen, Germany \\ ${ }^{3}$ University of Oxford, Department of Materials; Parks Road, Oxford OX1 3PH, UK
}

Keywords: Computer Model, Spray Forming, Inconel 718

\begin{abstract}
A unified computer model of the process of spray forming Inconel 718 rings has been developed. The target of the model is to optimize the process input parameters to achieve the correct shape, microstructure and minimum porosity. The model is divided in three main parts that correspond to the atomization phase of the material, the flight behaviour of the droplets and the solidification and growth of the ring. Different modelling techniques have been used for each part of the model. For the atomization step, stability analysis for the primary atomization and a tracking model for secondary atomization were used. Computational fluid dynamics calculations for the thermal and mechanical behaviour of the droplets in their travel to the substrate were used for the in-flight behaviour step. Shape and growth models, as well as thermal models, based on finite element calculations were used to predict the final shape and temperature history of the as sprayed ring. Various computer programs have been written to link results between different submodels and to transform the format of intermediate computer files.
\end{abstract}

\section{Introduction}

Spray forming is a known near-net shape process that is used for applications (aeronautical, automotive,...) where a refined equiaxed grain structure is important and where near-net shapes can be produced.. The alloy of interest in this study (Inconel 718) is melted in a crucible and converted into small droplets of different sizes with a pressurized gas flow jet. This atomization step is followed by the flight of the droplets towards the substrate. The material interchanges heat (through convection and radiation) and momentum with the surrounding gas. A process of rapid solidification is present in this step, with some of the droplets completely solid when arriving at the substrate and others in the semi-solid state or liquid. The final step is the deposition of these droplets and the incremental growth of the ring or billet. A photograph of the spray forming of a billet is shown in Figure 1.

Since the beginning of the utilization of the spray forming process - created by A.R.E. Singer in the 70 s of the last century - more than 30 years have passed and much attention has been devoted to the modeling and simulation of the process itself [1-6]. The complexity of this modeling is significant, as many different factors and phenomena take place in the spray forming process. Researchers have concentrated their efforts on a few submodels or parts of the whole process, assuming data coming from other researchers or studying in depth the influence of only a few parameters of the whole process. Although this approach is perfectly acceptable, a unified 
computer model of the spray forming process that captures all the important process physics may offer additional insights to optimize the process parameters, final microstructure, shape and economic performance.

1) Atomization

- Gas flow

- Metal flow

- Primary Atomization

- Secondary Atomization

2) Spray

- Gas Flow

- Droplet Flow

- Coupling

3) Deposition

- Droplet Impingement

- Shape Evolution

- Heat Flow

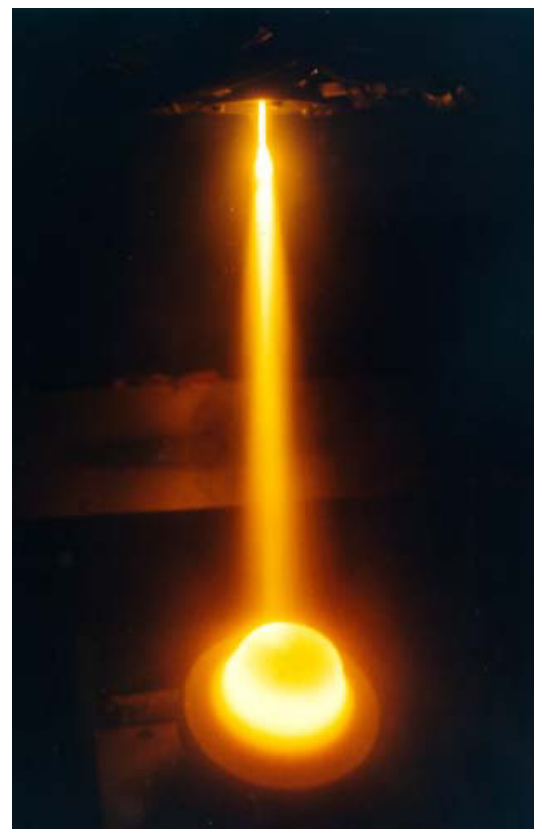

Figure 1. Spray forming process of a billet.

Many parameters can influence the final shape and properties of the billets and rings that can be produced with the spray forming process. Without being exhaustive, it is necessary to mention the material that is used, the mass of feed stock that is sprayed, the superheat that it is used over the temperature of melting, the geometry of the spray chamber, the nozzle diameter and its geometry, the employed gas $\left(\mathrm{N}_{2}\right.$, Ar), the melt flow rate and the gas flow rate, the scanning angle and scanning frequency of the nozzle, the distance from the nozzle to the substrate, the temperature and movement of the substrate and its rotating frequency. It is clear that all these parameters have some influence on the characteristics of concern and that optimization of the process by simple trial and error methods can be time consuming, very expensive and offer no security in obtaining the targeted results. This paper describes a unified computer model that can help to understand the physics of the process and that can produce guidance to questions of the type "what if..." in choosing the settings of the many process parameters.

\section{Atomization model}

The atomization model consists of two parts, reflecting the most commonly assumed nature of the fragmentation process of a liquid in a twin-fluid atomizer, namely, the primary break-up process and the secondary break-up process. Only the key features are described here, with the reader directed elsewhere for further details.

\section{Primary Break-up}

A cylindrical fluid element in a quiescent gaseous atmosphere is unstable. Small perturbations of the surface will always grow. Initialisation of the liquid jet disintegration is described by the initial perturbation and the growth of surface waves at the liquid/gas interface. Instability itself is due to variations in the surface tension, leading to a local pressure distribution on the phase boundary dependent on the local liquid surface curvature. 
The boundary layer effects of the gas flow field stabilizes the wave growth and are taken into account as a damping effect on the perturbation pressure acting on the liquid jet surface.

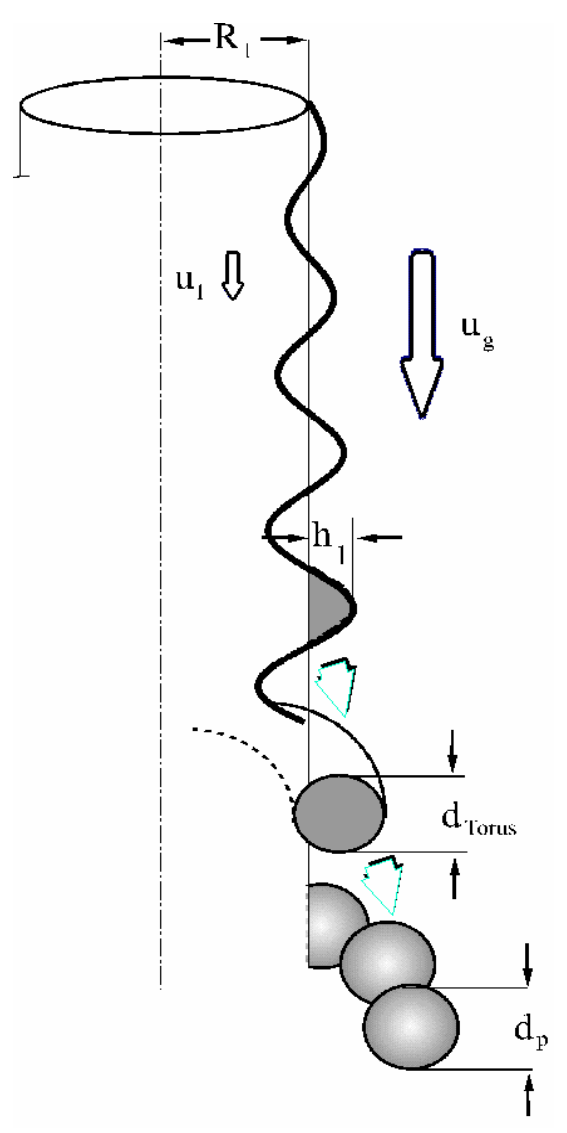

Figure 2: Primary break-up dynamics

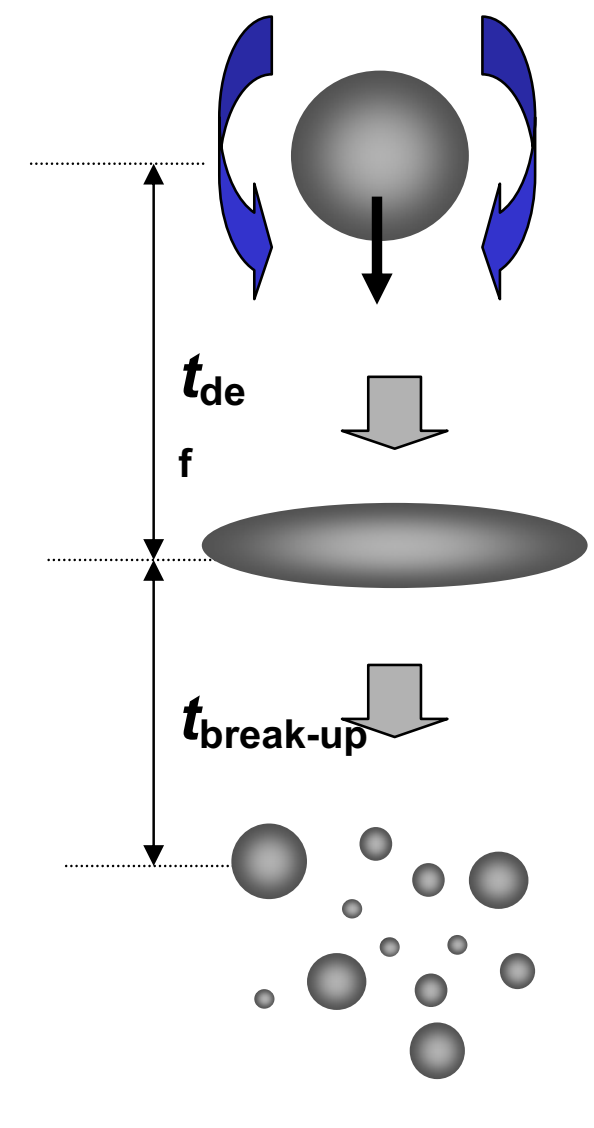

Figure 3: Secondary break-up dynamics

\section{$\underline{\text { Secondary Break-up }}$}

After primary break-up, the ligaments and droplets may further break-up into smaller droplets (secondary break-up) until the driving force for further break-up disappears. Simultaneously droplet collisions with further fragmentation or coalescence may occur. These processes finally result in the measured spray droplet size distribution. The secondary break-up is generally defined as the break-up of ligaments into droplets, including the successive break-up of larger, relatively unstable droplets into smaller droplets, as shown schematically in Figure 3.

A suitable description of the secondary break-up within the atomisation of metal melts for complex nozzle geometries needs to account for the bulk of all molten particles. Therefore, the break-up, the acceleration and the solidification/cooling of all particles, plus eventually the possible coalescence should be taken into account. For the simulations in this study, the gas velocity distribution within the atomization area was prescribed.

A classification of the different droplet break-up mechanisms in terms of the aerodynamic Weber number depends on the local relative velocity between the gas phase and the particle. Below a critical limit of the Weber number $\left(\mathrm{We}_{\text {crit }}<12\right)$ no further break-up occurs. A relatively well known description of this behaviour is given by Pilch \& Erdmann [7], which includes a description for high Weber numbers above We $\geq 350$. According to this classification, Samenfink [8] distinguished between three mechanisms for secondary break-up: 
Bag break up for $12<\mathrm{We} \leq 20$

Multi mode break up for $20<\mathrm{We} \leq 70$

Shear break up for $\mathrm{We} \geq 70$

The molten droplet deforms at first to a disk and then breaks-up into new droplets. For the computer model a Computational Fluid Dynamics (CFD) program has been used, and the domain is described by a 2-dimensional structured grid of 50 x 200 nodes. The computation is uncoupled (particles have no effect on the gas phase). The field variables are the mean velocity $u_{\text {gas }}, v_{\text {gas }}$, the kinetic energy $k$ and the gas temperature $T_{\text {gas. }}$ The turbulent velocity components $u_{\text {gas }}^{\prime}, v_{\text {gas }}$ are derived from random numbers. Each particle in the resulting spray is calculated. Droplet collision effects are ignored. The droplet dynamics and cooling/solidification have been calculated based on the models described in Bergmann [9]. The time step size used was $\Delta \mathrm{t}=10$ $\mu \mathrm{s}$ and 2500 iterations were calculated. It is assumed that the molten jet flows into the atomization zone at its original melting temperature within the tundish. Most particles will be produced in a short distance defining the region of primary break-up. The droplets are then accelerated and the spray cone diverges due to turbulence. Especially small particles may be undercooled significantly. As a result of these calculations, the information that it is obtained for each one of the particles is its diameter, temperature, position and velocity. For a typical calculation, (mass flow rate $0.4 \mathrm{~kg} / \mathrm{s}$ ) the number of particles can be as high as 16,000,000. Typical gas atomising conditions are shown in Table $\mathrm{I}$, where $\mathrm{P}_{\mathrm{o}}$ is the manometric pressure, $\mathrm{P}_{\mathrm{s}}$ is the static pressure and $\mathrm{P}_{\mathrm{op}}$ is the operational pressure. These gas conditions correspond to an ideal expanded flow for the primary atomizer and to a shocked flow for the secondary atomizer.

Table I: Atomising gas conditions

\begin{tabular}{|l|l|l|}
\hline & \multicolumn{2}{|c|}{} \\
\hline Atomizer & Primary & Secondary \\
\hline Material & \multicolumn{2}{|c|}{ IN 718 } \\
\hline Gas & $\mathrm{N}_{2}$ & $\mathrm{~N}_{2}$ \\
\hline $\mathrm{P}_{\mathrm{o}}$ (bar) & 1.5 & 4 \\
\hline $\mathrm{P}_{\mathrm{S}}$ (bar) & 0 & 1.6455 \\
\hline $\mathrm{P}_{\mathrm{op}}$ (bar) & 1 & 1 \\
\hline
\end{tabular}

The following figures show some results that are obtained in this atomization model. Figure 4 presents the cumulative number of particles (in percentage) that is obtained as a function of the particle size while Figure 5 indicates the cumulative mass of particles (in percentage) also as a function of the particle size. 


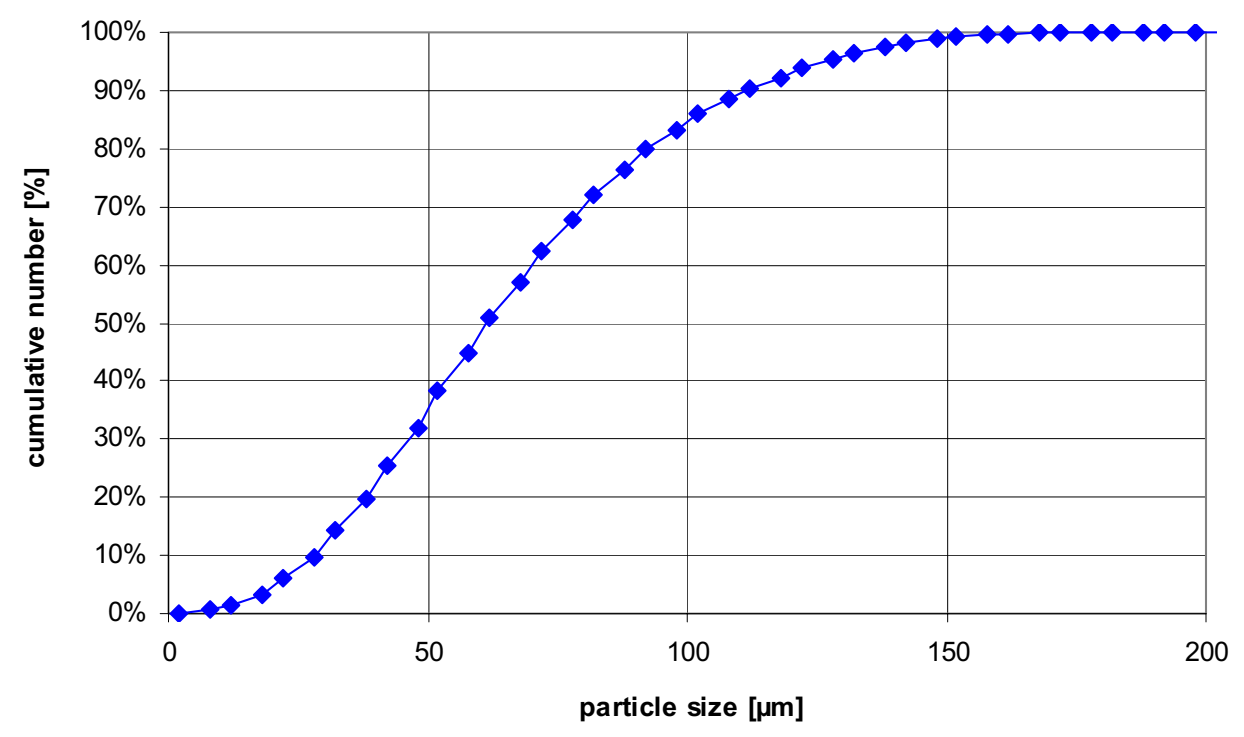

Figure 4: Cumulative number of particles as a function of particle size

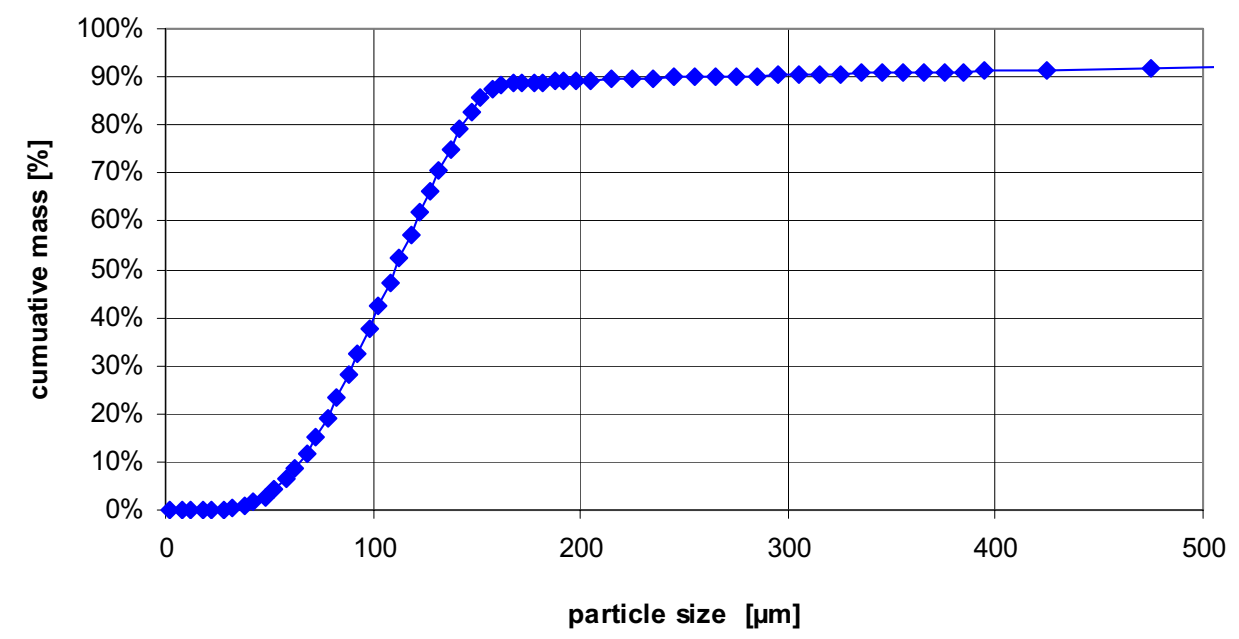

Figure 5: Cumulative mass of particles as a function of particle size

\section{In-flight behaviour of the spray}

Immediately following atomization, droplets are directed into or onto a shaped collector (mould or substrate). The droplets travel a predetermined distance prior to impingement on a deposition surface. The trajectory of the droplets may be predicted by integrating the drag force acting on the particles arising from the differential velocity between droplets and atomizing gas.

There are three separate forces that must be considered in calculating the velocity history of a droplet: a gravitational force, $F_{g}$, a fluid drag force, $F_{d}$, and a buoyant force, $F_{b}$. these forces are combined into a formulation of Newton's Second Law as follows:

$$
m_{d} \cdot \frac{d V_{d}}{d t}=F_{g}+F_{d}-F_{b}
$$

The simulation code used for the in-flight behavior part is FLUENT. In addition to solving transport equations for the continuous (gas) phase, the program allows the simulation of a 
discrete second phase in a Lagrangian frame of reference. This second phase consists of spherical particles (which represent the droplets) dispersed in the continuous phase. FLUENT computes the trajectories of these discrete phase entities, as well as heat and mass transfer to/from them. It is possible to include a discrete phase in the model by defining the initial position, velocity, size, mass and temperature of individual particles, and this information comes from the previously described atomization model step, and which is preprocessed with an inhouse developed program. These initial conditions, along with other inputs defining the physical properties of the discrete phase, are used to initiate trajectory and heat/mass transfer calculations. The trajectory and heat/mass transfer calculations are based on the force balance on the particle and on the convective/radiative heat transfer from the particle, using the local continuous phase conditions as the particle moves through the flow.

For the mechanics of the calculations itself, first of all, the gas settings have to be reproduced. Thus, gas properties and boundary conditions must be introduced into the FLUENT model and once the gas flow is simulated and the single phase flow field in the chamber has converged, the droplets are "injected" into the flow.

The discrete-phase injections are read by FLUENT from a specific file type from the atomization model. In a preliminary step, only a limited number of particles $(10,000)$ are introduced into the flow to maintain the calculation within reasonable CPU times. However, recent results in the third part of the model (deposition and growth model) have shown that this number of particles is not always sufficient to avoid numerical problems in the growth and deposition model, so that up to $6,000,000$ particles have also been considered, leading to long computation times, but improved results.

The main task to link the models together is to distribute droplets in the flow according to the atomization results, such as number of particles, mass flow rate, axial velocity, radial velocity, temperature, diameter and position. The success of this mapping process is crucial to provide sensible input data for the in-flight behavior model.

The material and gas data used in the simulations are given in Table II and III. As a result of the simulation, information about the position, velocity and temperature for each particle is obtained for different distances from the atomizer.

Table II: Inconel 718 properties used in simulations.

Table III: Atomizing gas properties.

\begin{tabular}{|c|c|c|}
\hline Superalloy properties & & IN718 \\
\hline Temperature (liquidus) $\left[{ }^{\circ} \mathrm{C}\right]$ & $\mathrm{T}_{\mathrm{L}}$ & 1336 \\
\hline Temperature (solidus) $\left[{ }^{\circ} \mathrm{C}\right]$ & $\mathrm{T}_{\mathrm{S}}$ & 1170 \\
\hline Density (liquidus) $\left[\mathrm{kg} / \mathrm{m}^{3}\right]$ & $\rho$ & 7027 \\
\hline Viscosity [kg/m s] & $\mu$ & 0.0075 \\
\hline Heat capacity (liquidus) $[\mathrm{J} / \mathrm{kg} \mathrm{K}]$ & $\mathrm{C}_{\mathrm{p}}, \mathrm{I}$ & 720 \\
\hline Heat capacity (solidus) [J/kg K] & $\mathrm{C}_{\mathrm{p}, \mathrm{S}}$ & 650 \\
\hline Latent heat [J/kg] & $\mathrm{L}$ & 210000 \\
\hline Equivalent heat capacity [J/kg·K] & $\mathrm{C}_{\mathrm{p}, \mathrm{e}}$ & $(1-\beta) \mathrm{C}_{\mathrm{p}, \mathrm{s}}+\beta \mathrm{C}_{\mathrm{p}, \mathrm{I}}+\mathrm{L} /\left(\mathrm{T}_{\mathrm{L}}-\mathrm{T}_{\mathrm{S}}\right)$ \\
\hline Surface tension $[\mathrm{N} / \mathrm{m}]$ & $\sigma$ & 1.882 \\
\hline Emissivity [unitless] & $\varepsilon$ & 0.7 \\
\hline
\end{tabular}

\begin{tabular}{|c|c|c|}
\hline Gas properties & Nitrogen & Argon \\
\hline Molecular weight & 28.02 & 39.944 \\
\hline $\mathrm{R}\left(\mathrm{m}^{2} / \mathrm{s}^{2}-\mathrm{K}\right)$ & 296.717 & 208.141 \\
\hline $\mathrm{Cp}(\mathrm{J} / \mathrm{kg}-\mathrm{K})$ & 1041 & 520 \\
\hline Thermal conductivity $(\mathrm{W} / \mathrm{m}-\mathrm{K})$ & 0.0243 & 0.01772 \\
\hline Density & \multicolumn{2}{|c|}{$\rho=\mathrm{P} /(\mathrm{R} \cdot \mathrm{T})$} \\
\hline
\end{tabular}

Similarly to output of the atomization model, output data from the in-flight model must also be post-processed into a sensible and useful format. Accordingly, this is achieved using another inhouse written code. The output file is a multi-list of properties such as mass flow rate, number of particles, velocities, temperature, etc, as a function of position. 


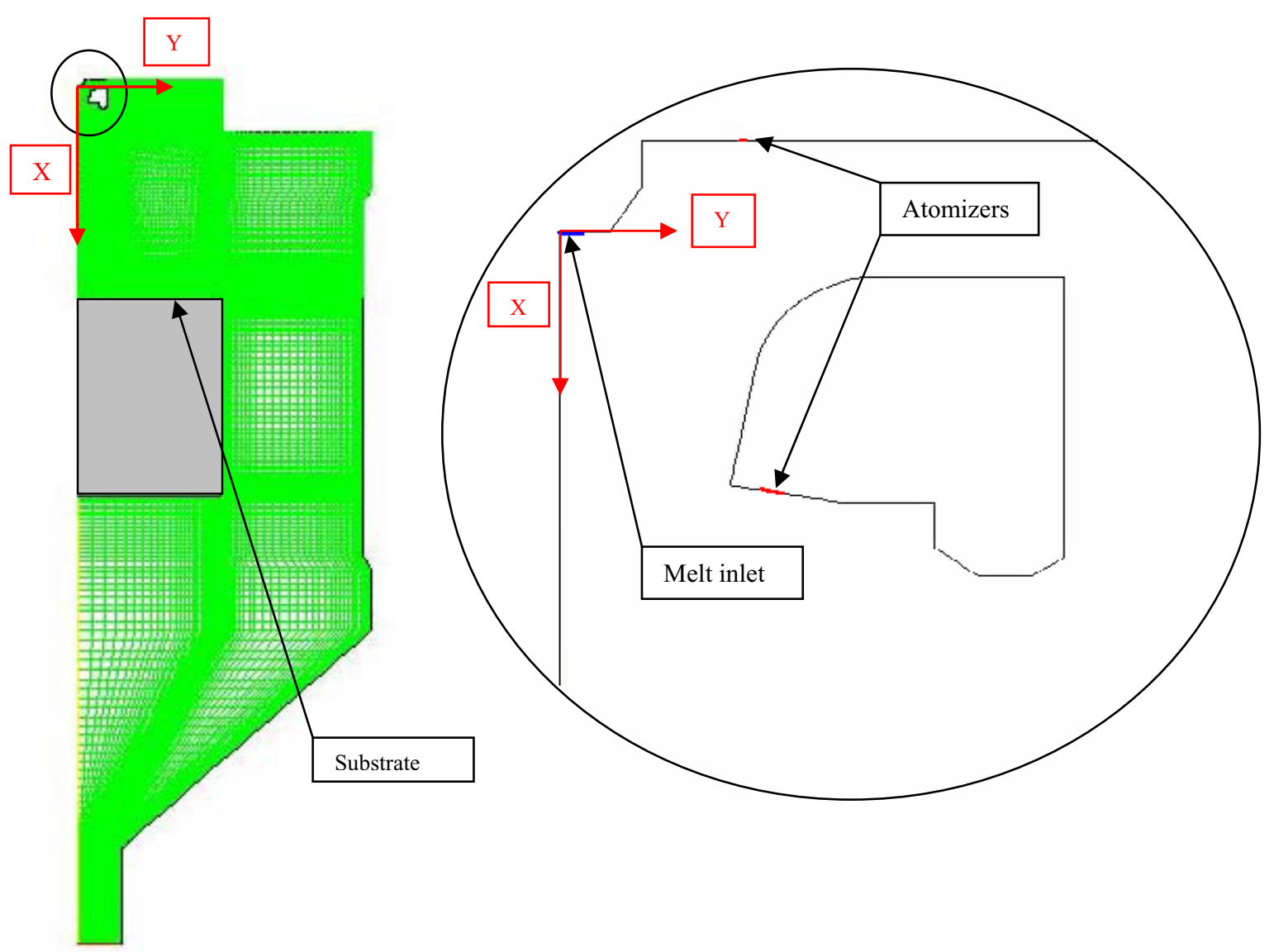

Figure 6: Mesh and geometry model of the chamber. Revolution symmetry around $\mathrm{X}$ axis.

Figure 6 shows the geometry of the spray chamber employed in this investigation and the mesh that has been used for the FLUENT simulations. In some regions, it is not possible to observe all the details of this mesh, because of the very small size of some of the elements.

\section{Deposition, shape evolution and heat transfer model}

In experiments to produce preforms of sufficient size, the secondary atomizer scans the spray with a pre-determined angle and frequency $\left(8.5^{\circ}\right.$ to $10.5^{\circ}$ and $\left.15.2 \mathrm{~Hz}\right)$ and this effect has to be taken into account for a proper estimation of the shape evolution of the deposit. In contrast, the data from the in-flight model is essentially steady-state, for a time-averaged 'static' spray. Therefore, the output data from the postprocessed FLUENT calculations must be transformed in order to reproduce the scanning effect. Hence, a third in-house developed code has been written to calculate the time-averaged mass flux and the enthalpy flux as a function of the position over one scan of the atomizer. The program also calculates the average deposited mass flow in one rotation of a ring substrate at a rotation velocity of $1.2 \mathrm{~Hz}$.

Figure 7 shows a typical deposition rate (m/cycle) and impinging spray temperature distribution $\left({ }^{\circ} \mathrm{C}\right)$ including atomizer scan and substrate rotation effects. The maximum growth rate (deposition rate) is approximately $4.38 \times 10^{-4} \mathrm{~m} /$ cycle and the profile has a double peak shape associated with particular scan pattern employed. Temperatures are relatively flat at $\sim 1292{ }^{\circ} \mathrm{C}$. The two extreme temperature peaks at a distance of $\sim 0.12 \mathrm{~m}$ from the center-line correspond to numerical problems at the ring edge, attributed to the averaging method. 


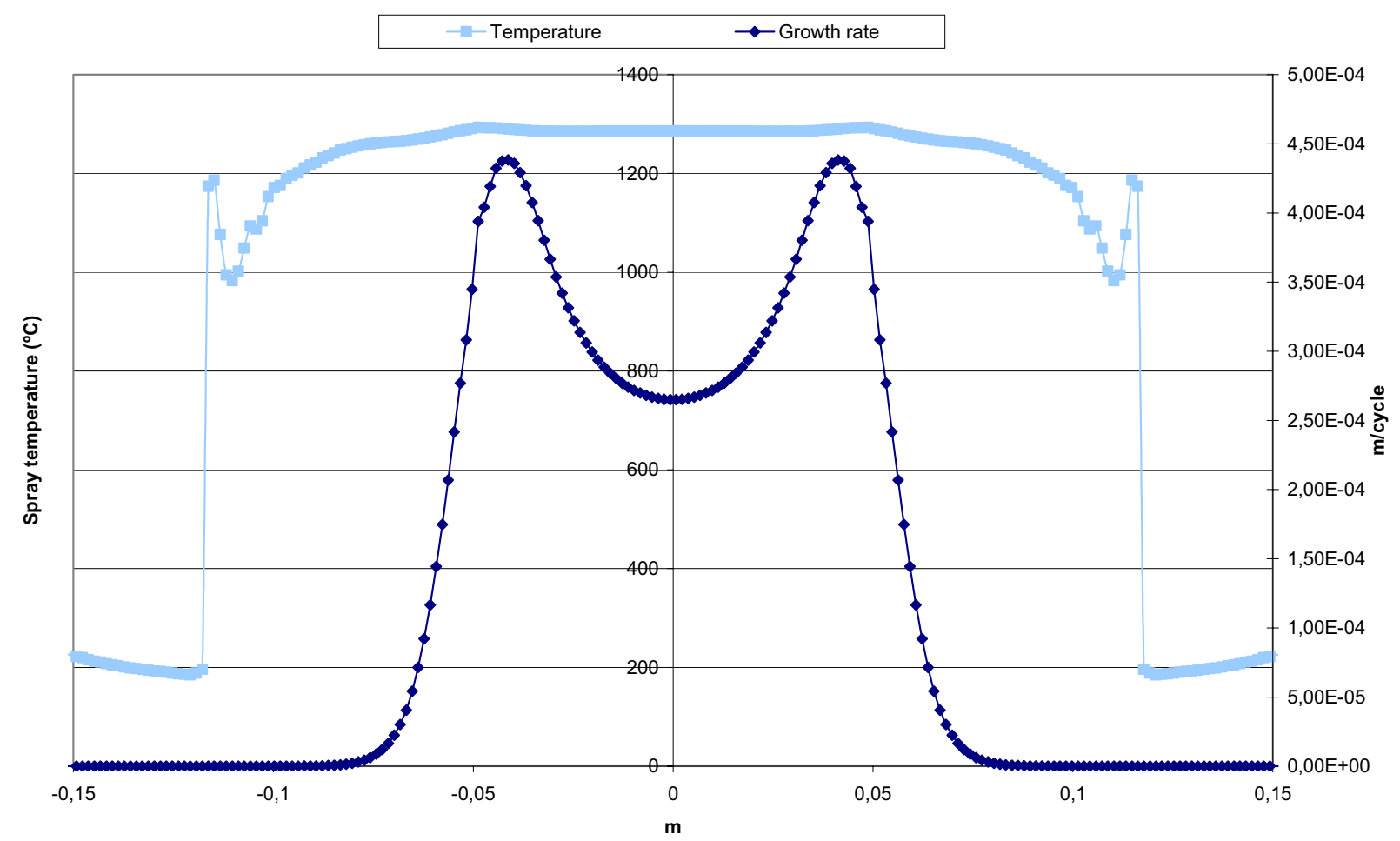

Figure 7: Growth rate and temperature on average on the deposit of an Inconel 718 ring

With this input, it is now necessary to simulate the growth of a ring-shaped deposit on a tubular substrate for each rotation of the substrate, including the heat transfer to the substrate and chamber environment, and also accounting for any lateral movement (withdrawal) of the substrate in the axial direction. To do this, a completely new Matlab/Femlab-based code has been developed. The code uses cubic spline technique to calculate the dynamic growth of the deposit shape over every substrate rotation and Finite Element Method to solve the heat transfer equations. The algorithm implemented to tackle the coupling of deposit shape and thermal dynamic comprises steps of: (1) define a thin region just below the top surface of a deposit; (2) calculate and update the top surface to the new position when materials are deposited; (3) stretch the mesh points of the original top surface to the new positions and therefore account for the new mass input; (4) recalculate the heat in the thin region to account for heat input and ensure the energy conservation; (5) perform heat transfer calculation for the new deposit and the substrate; (6) remesh the new deposit and map all the data onto the new mesh structure; (6) restart step 1.

$\underline{\text { Shape evolution model }}$

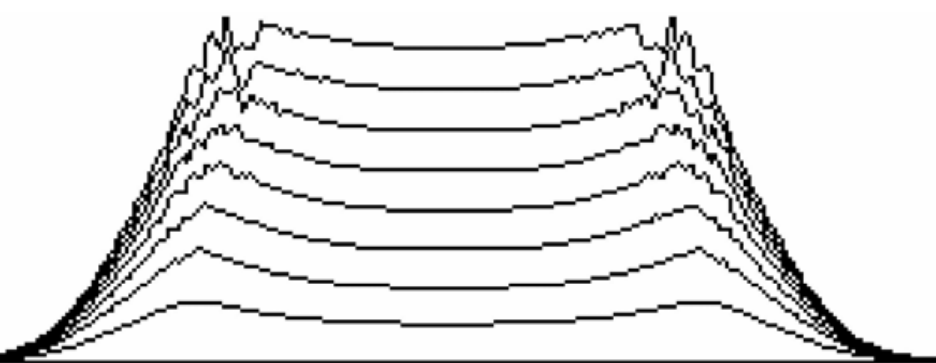

Figure 8: The calculated ring cross-section shape under typical conditions, after 30 substrate revolutions. 


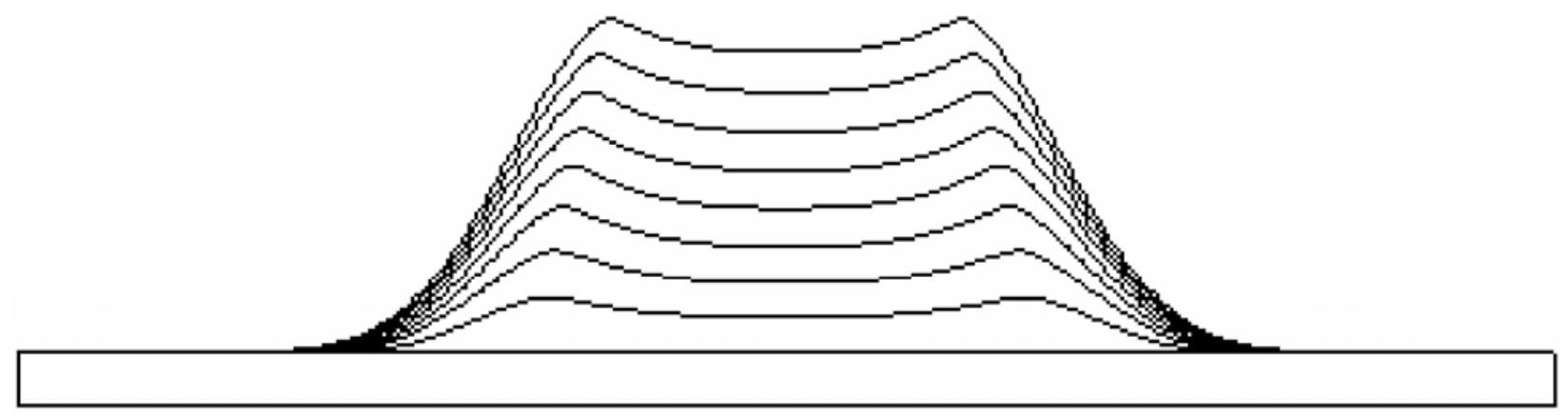

Figure 9: The calculated ring cross-section shape for the same conditions as Figure 8, but with data smoothing.

The integration in time of the deposited mass calculated from the previous deposit shape plus the new mass arrived in a small time-step using the data in Figure 7 led to some numerical stability problems when several layers of material were added. Figure 8 shows the preform shape that is obtained by directly integrating the mass flow and Figure 9 shows the shape that is obtained when a double smoothing operation is performed. Small numerical irregularities in the initial data are amplified through multiple integrations and smoothing of data (making sure that mass is conserved) is needed.

The final predicted shape is obtained after around 400 revolutions (the whole spray process) and also taking into account the sticking efficiency of the spray as a function of deposition angle, where a linear relation was assumed between $75 \%$ deposition for $0^{\circ}$ (perpendicular impingement) and $0 \%$ deposition for $90^{\circ}$ (parallel impingement). The predicted ring cross-sectioned final shape compared with the experimental final shape of a section of rings sprayed under three different conditions are shown in Figure 10, for the full interlinking of all three sub-models. In each case, the central zone of the predicted shape matches reasonably well with the real shape. The area further to the left or right of the ring is less well matched because the secondary deposition of that fraction of mass non-deposited on the initial, primary impact has not been taken into account. It is also likely that a temperature dependency of sticking efficiency should be included. Nonetheless, the part of the ring that will be used for components is the central part, and in these regions the model provides good predictions over a range of conditions. Further developments to model the 'shoulder' regions are underway.

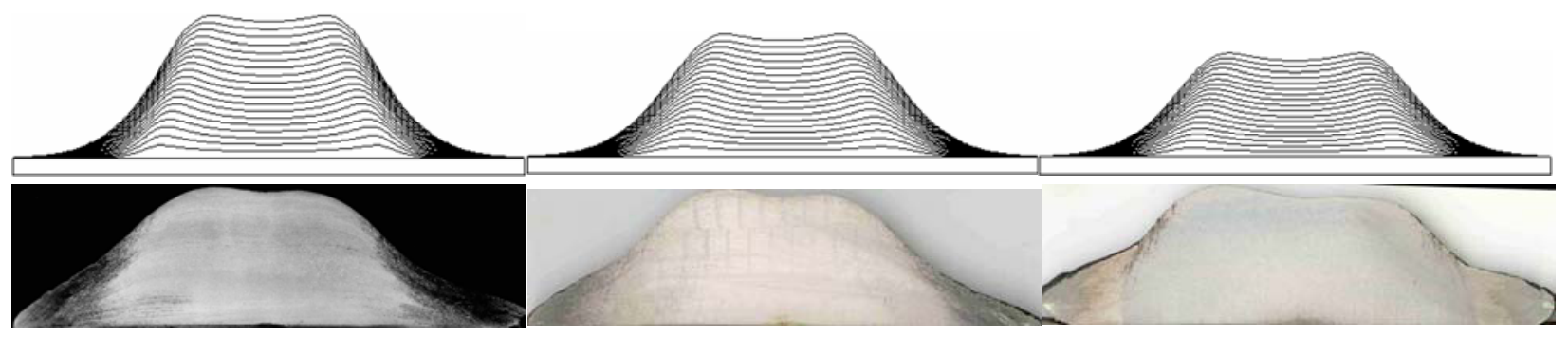

Figure 10: IN718 Predicted (upper) and experimental (lower) ring cross-sectional shapes for three different spray forming conditions. 


\section{$\underline{\text { Heat flow model }}$}

The deposit heat flow model runs in parallel with the shape evolution model and accounts for the conduction, convection and radiation phenomena that occur in the deposit and between the deposited material and the substrate/surrounding environment. The precise values of some of the different parameters required (convection coefficients, emissivity) are difficult to simulate, but sensitivity studies in other spray forming studies have identified sensible values that also show good agreement with experiment when comparing simulation results with measured temperatures [10]. A typical mesh for the heat flow model at an instant in the deposition is shown in Figure 11, where the figure on the right shows how the mesh points of the original top surface are stretched to the new top surface to account the new mass input.

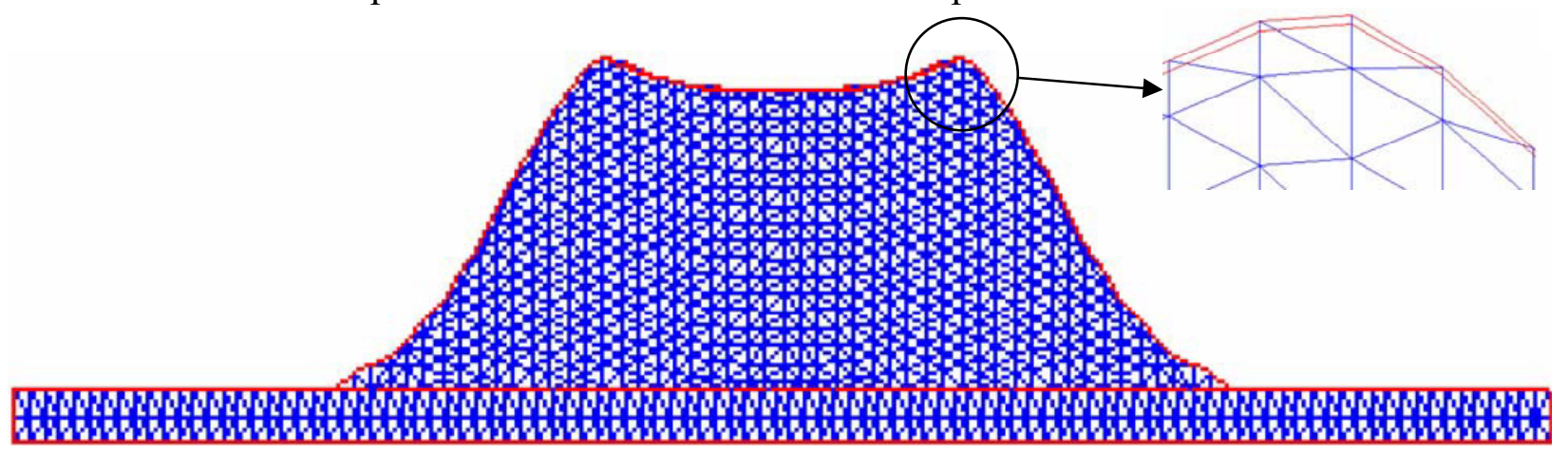

Figure 11: Deposit and substrate mesh for the heat flow model

The heat flow model provides temperature profiles and alloy liquid fraction contours as a function of time, and typical results are shown in Figure 12. The temperature evolution of deposit top surface region is clearly demonstrated. These macroscale temperature/time histories are now forming the basis for further sub-models to describe the porosity and grain size of spray formed alloys that are also under development.

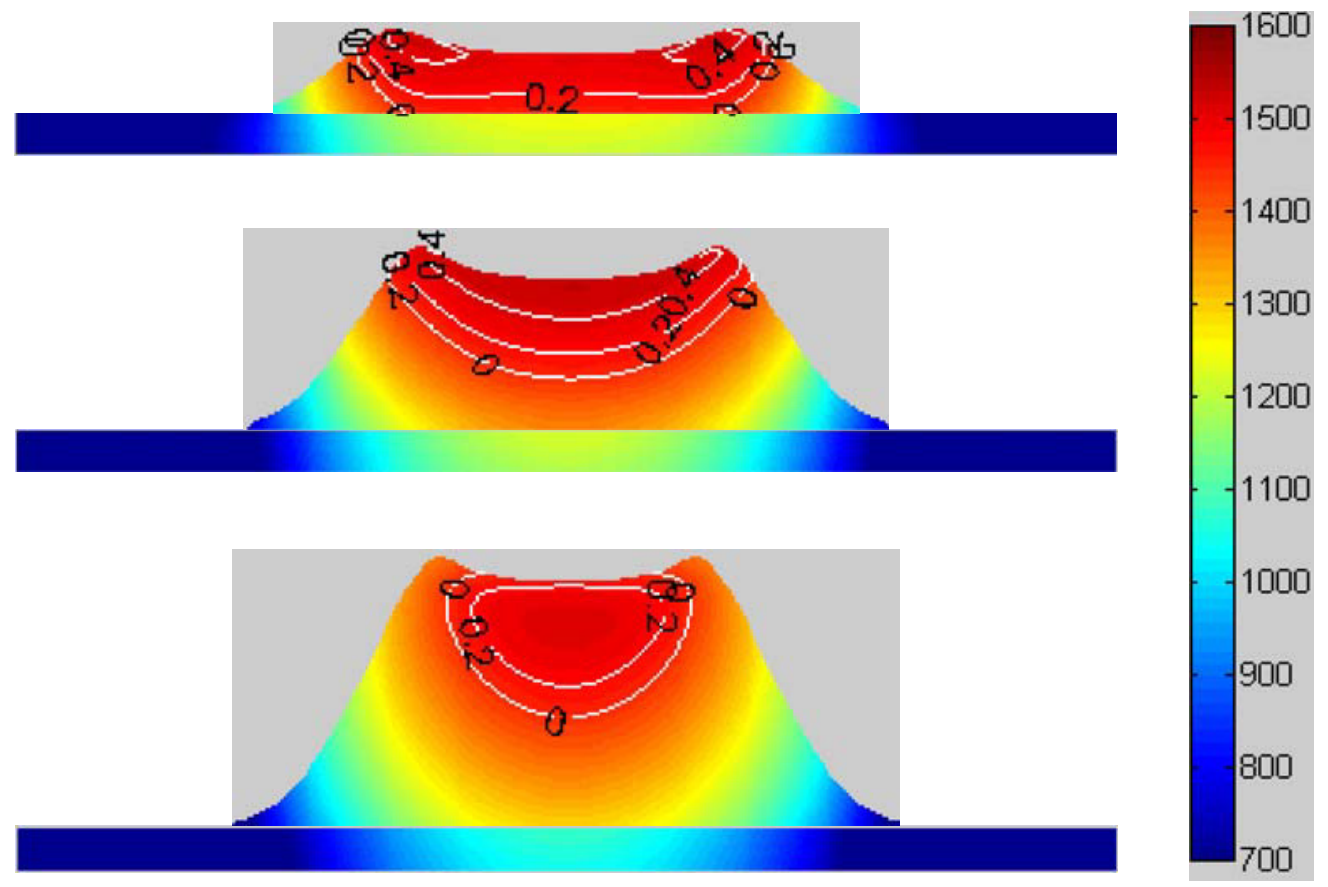

Figure 12: Temperature profile $(\mathrm{K})$ and liquid fraction contour in the cross-section of a typical ring deposit at three instants during spray forming. 


\section{Conclusions}

A unified model of the spray forming process of Inconel 718 rings has been developed that links together first principles calculations of the three key steps in spray forming: atomization; inflight behaviour of droplets; and deposition, shape evolution and heat flow. In all three models, assumptions are required regarding the underlying process physics and some critical parameters. Nonetheless, the unified model has captured well the key characteristics of spray formed IN718 ring geometry and thermal behaviour. Further refinements are underway to include secondary deposition, and to link these macroscale models to sub-models of as-sprayed porosity and grain size.

\section{Acknowledgments}

The support of the European Commission under the contract G4RD-CT2002-00762 is gratefully acknowledged. The financial help of the Department of Education, Basque Government, through its mobility program, MV-2004-1-3, for the stay of I. Garmendia at Oxford University is also gratefully acknowledged.

\section{References}

[1] E.J.Lavernia, Y.Wu, "Spray Atomization and Deposition”, Wiley, New York, NY 1996.

[2] U.Fritsching, "Modeling and Numerical Simulation of Sprayforming Metals", Cambridge University Press, Cambridge, UK, 2004.

[3] Shinill Kang, Dong-Hoon Chang, "Modelling of billet shapes in spray forming using a scanning atomizer", Materials Science and Engineering, A260 (1999) 161-169

[4] T.B.Pedersen, J.H.Hattel and N.H. Pryds, "A geometrical model for the prediction of the billet shape in spray forming”, Proc. $22^{\text {nd }}$ Int. Symp. On Material Science, Denmark 2001

[5] R.P.Underhill, P.S.Grant, B.Cantor and D.J.Bryant, "Modelling of droplet behaviour during spray forming using Fluent", International Journal of Non-Equilibrium Processing, 1997, Vol 10, pp 201-216.

[6] A.M.Sterling, C.A.Schleicher "The Instability of Capillary Jets". J. Fluid Mech. 68 (1975), $477-495$

[7] M.Pilch, C.A.Erdmann "Use of Break-up Time Data and Velocity History Data to Predict the Maximum Size of Stable Fragments for Acceleration-Induced Break-up of a Liquid Drop", Int. J. Multiphase Flow 13 (1987), 741-757

[8] W. Samenfink, A. Elsäßer, S. Wittig and K. Dullenkopf (1996): "Internal Transport Mechanism in Shear-Driven Liquid Films", $8^{\text {th }}$ international Symposium on Applications of Laser Techniques to Fluid Mechanics, Lisbon, Portugal

[9] D.Bergmann,U.Fritsching,K.Bauckhage "A mathematical model for cooling and rapid solidification of molten metal droplets", Int. J. Therm. Sci. 39 (2000), 53 - 62

[10] Z. Shi, J. Mi and P. S. Grant "Numerical heat transfer modeling in spray formed IN718 billets", Mat. Sci. Forum, 475-479 (2005), 2803-2806.

[10] J. Mi, Z. Shi, and P. S. Grant "Modelling shape evolution and heat flow of spray-formed ring preforms", Mat. Sci. Forum, 475-479 (2005), 2807-2810. 